\title{
El aprendizaje mixto en la preparación de la Prueba de Acceso a la Universidad en Lengua Inglesa: Aportaciones de un estudio experimental
}

\author{
Elena Martín Monje \\ $U N E D$
}

Recibido: 24 noviembre 2011 / Aceptado: 16 febrero de 2012

ISSN: $1697-7467$

\begin{abstract}
RESUMEN: El análisis de los datos de la prueba de acceso a la universidad en lengua inglesa de los últimos años evidencia unos bajos resultados de este idioma frente a los de otras materias, por lo que resulta propicia una intervención educativa que explore las causas y ofrezca soluciones en este ámbito. Este trabajo muestra los resultados de un estudio experimental en el que se introduce una nueva metodología de aprendizaje mixto, combinando enseñanza presencial con el apoyo de un entorno virtual de aprendizaje, con el objetivo de aumentar el tiempo de dedicación por parte del alumnado, su motivación al estudiar la lengua extranjera y conseguir una mejora en los resultados en un curso académico clave como es el previo a la universidad.

La investigación se enmarca dentro de la investigación-acción, tomando en cuenta las últimas propuestas metodológicas en enseñanza de lenguas -enfoque comunicativo y Marco Común Europeo de Referencia para las Lenguas- así como los postulados teóricos de la enseñanza de lenguas asistida por ordenador y la perspectiva constructivista de los entornos virtuales de aprendizaje como Moodle, la plataforma educativa elegida para el experimento.

Los datos obtenidos arrojan un panorama alentador en la integración de las tecnologías de la información y las comunicaciones en la enseñanza de idiomas y muestran lo acertado de una metodología innovadora como el aprendizaje mixto en la consecución de unos mejores resultados en la prueba de acceso a la universidad en lengua inglesa.

Palabras clave: Prueba de Acceso a la Universidad, lengua extranjera, Tecnologías de la Información y de la Comunicación, entorno virtual de aprendizaje, Moodle.
\end{abstract}

Blended learning for the preparation of the University Entrance Exam in English: Findings of an experimental study

ABSTRACT: The analysis of the data provided by the Spanish university entrance exam in the past few years shows low results in English as a foreign language when compared to other subjects. It is relevant, then, to do some research and propose a solution in the field. This paper shows the results of an experimental study in which a new blended learning methodology is put forward with the objective of improving the results and increasing the time that students devote to the subject and their motivation to study the foreign language in a key academic year for them, just before going to university.

With an action-research approach, the study comprises the latest methodological proposals in language teaching -communicative approach and Common European Framework of Reference for Languages- as well as the guidelines of Computer Assisted Language 
Learning and the constructivist theories behind the use of virtual learning environments such as Moodle, the software chosen for the experiment.

The information obtained presents a promising outlook for the integration of information and communication technologies and foreign languages and it shows how pertinent it is to implement blended learning as a new methodology in order to obtain better results in the university entrance examination in English as a foreign language.

Keywords: university entrance exam, foreign languages, Information and Communication Technologies, virtual learning environment, Moodle.

\section{INTRODUCCIÓN}

En este artículo se presenta un estudio experimental sobre la utilidad de los entornos virtuales de aprendizaje (EVA) para la mejora de los resultados y el aumento en la motivación y dedicación de los estudiantes de $2^{\circ}$ Bachillerato que preparan la Prueba de Acceso a la Universidad (PAU) en lengua inglesa ${ }^{1}$. En la época de cambio e incertidumbre en que se encuentra la PAU en lengua extranjera, en que se espera la introducción de una prueba oral que nunca termina de llegar, se analizan las posibilidades que ofrecen los EVA en el área de lenguas extranjeras en general, y en la lengua inglesa en particular, con la finalidad de obtener una mejora en los resultados en un curso clave para su futuro académico, mediante la combinación de enseñanza presencial y aprendizaje en línea.

La realización de trabajos extraescolares de refuerzo y extensión es una práctica educativa común en todas las asignaturas, incluida la lengua inglesa. En la etapa de Bachillerato estas actividades fuera del horario escolar suelen centrarse en ejercicios de comprensión y expresión escrita, puesto que el tipo de PAU en vigencia en el curso escolar en que tuvo lugar el experimento (2008-2009), e incluso en la fecha de elaboración de este artículo, se ocupa de estas dos destrezas exclusivamente (BOE núm. 238, de 4 de octubre de 1990). Lo novedoso en este experimento ha sido la metodología utilizada: se optó por el aprendizaje mixto (AM), que combinaba las clases presenciales con la ayuda de un EVA en el que se proponían actividades de apoyo a la asignatura con una periodicidad semanal (práctica de gramática y exámenes de años anteriores, creación de un glosario personal, actividades de comprensión y producción escrita, actividades de comprensión, producción e interacción oral, técnicas de examen, etc.). A continuación se profundiza en el marco teórico en el que se desarrolla la investigación.

\section{Marco teórico}

La perspectiva teórica adoptada se basa en tres pilares fundamentales: investigaciónacción como metodología investigadora, enseñanza de lenguas extranjeras y enseñanza asistida por ordenador. La investigación-acción (IA) resulta pertinente en este caso por la duplicidad

\footnotetext{
${ }^{1}$ La autora quisiera expresar su agradecimiento al grupo de investigación ATLAS (Artificial Intelligent Techniques for Linguistic Applications), con $\mathrm{n}^{\circ}$ de referencia 87H31, y el proyecto I-AGENT (Tutor Genérico de Inglés Ingeligente y Adaptativo), financiado por el Ministerio de Ciencia e Innovación ( $\mathrm{n}^{\mathrm{o}}$ de subvención FFI2008-06030) en cuyo seno se ha gestado la presente investigación.
} 
en el rol del investigador, que además es participante en el proceso. Se une así el conocimiento teórico con el de un contexto determinado y se procede al estudio de una situación educativa concreta con la intención de intervenir y mejorar dicha situación (Altrichter, Posch y Somekh, 1993; Cohen y Manion, 2004; Latorre, 2003; Palacios, 2007). Iniciada por Kurt Lewin en el siglo pasado con el objetivo de modificar las costumbres de los ciudadanos, la IA tiene como rasgo distintivo la combinación de teoría y práctica, observación e intervención (Buendía, 1999; Latorre, Rincón y Arnal, 1996; Masters, 1995) y en el contexto educativo es común que el investigador sea también el profesor (Latorre, 2003). Suele partir de un problema y profundizar en él aplicando el método científico, desde la definición del problema por investigar hasta el análisis de los resultados. Así pues, el ámbito de la IA es muy amplio, ya que comprende desde la metodología individual de un determinado profesor, hasta en ocasiones cambios a nivel institucional y global en la educación.

En alguna ocasión se ha cuestionado la objetividad de esta modalidad de investigación: el hecho de estar el profesor-investigador implicado en la situación investigada podría condicionar los resultados (Elliot, 1997; Suárez, 2002). Sin embargo, los autores que trabajan en la línea de la IA la defienden alegando que la objetividad es más propia de la investigación del mundo físico-natural, mientras que en contextos sociales son más importantes otros aspectos epistemológicos, como el conocimiento crítico (Latorre, 2003; Suárez, 2002); la validez interna de la IA queda garantizada por la aplicación de procesos globales en la investigación, valiéndose de una triangulación de datos, y también ante la evidencia de las transformaciones reales producidas tras la intervención del investigador.

En cuanto a la enseñanza de lenguas extranjeras, es fundamental tomar como referencia tanto el enfoque comunicativo, en uso desde la segunda mitad del siglo XX, como el enfoque orientado a la acción propuesto en el Marco Común Europeo de Referencia para las Lenguas (MCER), de aparición más reciente (Consejo de Europa, 2002). Fundamentado en la sociolingüística y la lingüística funcional, el enfoque comunicativo ha sido hasta hace pocos años la tendencia metodológica más popular en la enseñanza y aprendizaje de idiomas (Richards y Rodgers, 2001; Sánchez Pérez, 2000; Varela, 2003). Según esta perspectiva, la lengua es un sistema de expresión de significado, por lo que su función primordial es la interacción y la comunicación. De este modo, las tareas y el lenguaje utilizado han de ser significativos para el alumno y facilitar su aprendizaje (Nunan, 2000; Palacios, 2007). Hoy en día sigue en vigencia, con numerosos manuales publicados según sus principios, y con influencia en otros métodos y enfoques, como el orientado a la acción, piedra angular del MCER. Este énfasis en la acción en la práctica de la lengua es uno de sus aspectos más novedosos, ya que se plantea el aprendizaje de un idioma como un acto social que nos ayuda a llevar a cabo determinadas tareas, tanto lingüísticas como no lingüísticas. La lengua es concebida primordialmente como un instrumento de comunicación (Fernández López, 2003), siguiendo la estela del enfoque comunicativo, pero orientada hacia la acción y caracterizando a los alumnos y usuarios de una lengua como agentes sociales, "miembros de una sociedad que tiene tareas (no sólo relacionadas con la lengua) que llevar a cabo" (Consejo de Europa, 2002: 9).

Es además necesario mencionar la enseñanza asistida por ordenador (EAO) y más específicamente la centrada en la enseñanza de lenguas o ELAO. Desde las últimas décadas del siglo XX, la aparición de Internet posibilitó nuevas metodologías en el proceso enseñanza-aprendizaje como el e-learning, aprendizaje a distancia por ordenador, o la mezcla 
de enseñanza presencial y teleformación (blended learning). Esta última metodología ha sido la preferida por los profesores de idiomas desde los comienzos de la ELAO (Neumeier, 2005), puesto que aúna los beneficios del aprendizaje en línea y la enseñanza tradicional, y ha sido la elegida por la autora en la aplicación del nuevo método instructivo dentro de la investigación que nos ocupa.

Se han ofrecido multitud de definiciones para este AM (Barret y Sharma, 2007; Sharma, 2010), pero quizá la más completa es la propuesta por Claypole (2010), para quien el AM es una metodología según la cual el profesor prepara una clase presencial y además tareas o actividades en un EVA que complementan y expanden lo aprendido en la clase, proponiendo de este modo el uso integrado de técnicas de enseñanza humanas y electrónicas, adaptadas según las necesidades del alumno y las capacidades del profesor. Con esta metodología el aprendizaje en línea y la formación presencial se complementan y enriquecen mutuamente, tal y como se puede apreciar en la figura 1 :

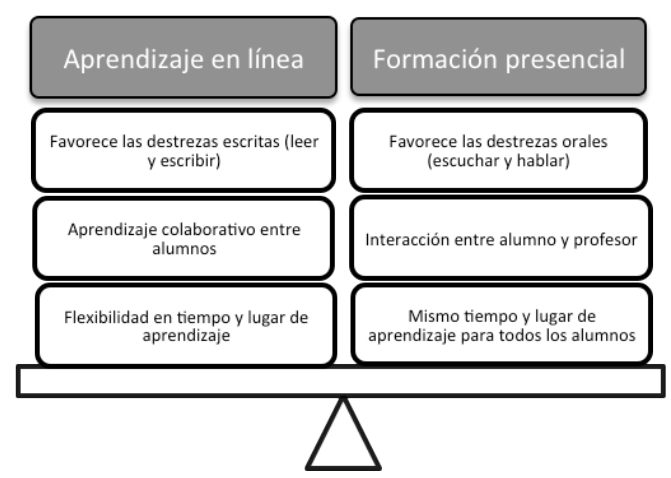

Figura 1. Equilibrio entre aprendizaje en línea y formación presencial.

Internet se convierte de este modo en un espacio de aprendizaje, tal y como hasta ahora lo había sido el aula, en el que el profesor debe favorecer que el alumno tome un papel activo (Blake y Standish, 2000; Cabero, 2006). Este nuevo espacio estimula la creación de nuevas formas de comunicación y relación, la interactividad y el aumento de la autonomía del alumno respecto a su aprendizaje (Salinas, 2008). En los EVA los alumnos tienen acceso a numerosos recursos en línea, herramientas de comunicación (correo electrónico o mensajería instantánea) o herramientas de trabajo colaborativo (foros, blogs, wikis), todo ello concebido desde una perspectiva constructivista del aprendizaje, enfoque clave en los EVA en general y en Moodle en particular, ya que parte de la premisa de que los alumnos construyen su propio conocimiento mediante la interpretación de su experiencia personal de aprendizaje y sus creencias y conocimientos previos (Dougiamas, 1998); en palabras de Carretero (1993: 21) "el conocimiento no es una copia de la realidad, sino una construcción del ser humano".

Esta pedagogía es necesaria para comprender el trasfondo teórico de la presente investigación, en la que se desarrolló un aula virtual de inglés utilizando el software libre de Moodle, de modo que los alumnos tuvieran acceso a materiales de apoyo a lo aprendido en la clase 
presencial. No es la primera vez que se investiga la utilidad de Moodle en el aprendizaje de idiomas (Brandl, 2005). Una de las iniciativas más interesantes es el proyecto COVCELL, coordinado por la Universidad de Islandia, www.covcell.org. Sin embargo, hasta ahora dicha investigación ha estado focalizada en la etapa universitaria y apenas se ha profundizado en las enseñanzas medias. Resulta, pues, oportuno el abordar dicha investigación en el contexto español, en que la prueba de idiomas en la PAU se encuentra en plena evolución. En el apartado siguiente se procede a la descripción del experimento.

\section{Desarkollo del experimento}

\subsection{Introducción}

El punto de partida del experimento fue, tal y como se ha expuesto anteriormente, la observación de ciertas carencias en lengua inglesa por parte de los alumnos de Bachillerato que se presentan a la PAU. La carga lectiva en los dos cursos de Bachillerato de tres horas semanales de lengua extranjera (BOE núm. 106, de 4 de mayo de 2006), resulta insuficiente para una correcta preparación de la asignatura. Esta circunstancia redunda en unos bajos resultados en la PAU de lengua inglesa en relación a las otras materias incluidas en esta prueba. En la Universidad Politécnica de Madrid, por ejemplo, una de las seis universidades en que se realiza la PAU en la Comunidad de Madrid (y la universidad a la que está adscrito el centro en que se realizó el experimento), al analizar los datos de la PAU de 2007, última convocatoria antes de comenzar el estudio experimental, se observa un claro desfase entre los resultados generales y los de la prueba en lengua inglesa. En ese curso escolar aprobaron en la convocatoria de Junio el $80,76 \%$ de los alumnos presentados; sin embargo, en lengua inglesa solamente pasaron el examen el 67,36\%, un 13,40\% menos. Estos malos resultados se corroboran y extienden por la geografía española, y son causa de preocupación entre estudiantes y docentes, como prueban varios titulares encontrados en prensa en los últimos años: en el diario Levante - El mercantil valenciano, "Los estudiantes llegarán a la prueba de selectivo de inglés sin dominar el idioma" (Ducajú, 2009) o en El Correo digital "El inglés estropea los buenos resultados de los alumnos riojanos en Selectividad" (Muñoz, 2009). Ante semejante situación, resultó de interés para la investigadora poner en marcha un programa de intervención didáctica acorde con los nuevos tiempos y las innovaciones pedagógicas que proporcionan las nuevas tecnologías y los EVA, que lograra una mejor preparación de los alumnos al enfrentarse a la prueba de inglés de la PAU, al mismo tiempo que les motivaba para que dedicaran un mayor tiempo de estudio a la lengua extranjera.

Este objetivo principal se puede desglosar en otros más específicos, explicados a continuación y descritos de forma gráfica en la figura 2 :

- Creación de un Aula Virtual de Inglés para Bachillerato utilizando la plataforma Moodle, ofrecida por el portal educativo EducaMadrid en los centros educativos de la Comunidad de Madrid, así como actividades interactivas -especialmente modelos de examen tipo PAU que sean susceptibles de ser calificados de modo electrónico.

- Aumento del tiempo de exposición a la lengua inglesa (o mayor input) en Bachillerato. 
- Práctica extraescolar de diferentes aspectos de la competencia lingüística, según el MCER: escuchar, leer, conversar, hablar, escribir (con especial hincapié en las actividades de comprensión y expresión escrita, puesto que son las únicas que se evalúan en la PAU por el momento).

- Seguimiento del progreso del alumnado utilizando EVA.

- Entrenamiento en la competencia digital, mediante la introducción de las tecnologías de la información y las comunicaciones (TIC) en la práctica docente.

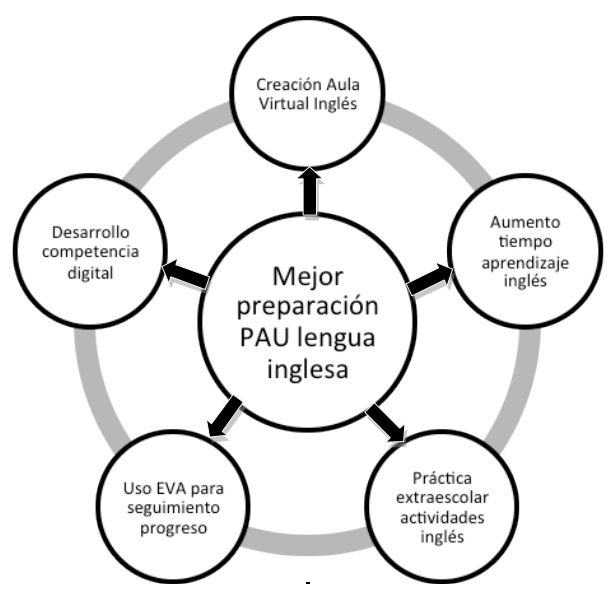

Figura 2. Objetivos de la investigación.

Una vez identificado el objeto de estudio, se procedió al desarrollo del plan estratégico de actuación completo:

1. En el primer paso (ya mencionado) se realizó la identificación y diagnóstico del problema: tres horas semanales de lengua inglesa no son suficientes para la preparación de la PAU, lo cual conlleva peores resultados en inglés que en otras materias.

2. En el segundo paso se formuló la hipótesis de investigación: el cambio de metodología, mediante la utilización de los EVA, da la posibilidad de ampliar el horario lectivo de la asignatura de lengua inglesa, ya que los materiales y actividades están disponibles al margen de la clase presencial. Se preveía que con este tratamiento instructivo aumentara el tiempo dedicado a la asignatura por parte del alumno y mejoraran sus resultados académicos, logrando superar la PAU más fácilmente.

3. En el tercer paso se planificaron las estrategias a seguir: se creó un Aula Virtual de Lengua Inglesa utilizando la plataforma Moodle ofrecida por el portal educativo EducaMadrid, así como las actividades pertinentes, de acuerdo con el currículo de Bachillerato y la programación de la asignatura.

4. El cuarto paso consistió en la aplicación del tratamiento instructivo, realizado durante todo el curso escolar 2008-09 con uno de los grupos de $2^{\circ}$ Bachillerato del Colegio Los Tilos en Madrid: utilización del método innovador con el grupo experimental, mientras que el grupo de control continuaba con la metodología tradicional. 
5. El quinto paso radicó en la recogida de datos, mediante procedimientos cuantitativos (tests, pruebas objetivas y exámenes) y cualitativos (cuestionarios, diarios, portfolio, observaciones, foros, entrevistas, etc.).

6. A continuación se realizó el análisis e interpretación de los datos, con ayuda del programa estadístico Statistical Package for Social Sciences (SPSS).

7. Una vez analizados los datos tuvo lugar la refutación o confirmación de la hipótesis planteada al comienzo de la investigación.

8. El último paso fue la elaboración de conclusiones, incluyendo una reflexión sobre los pasos seguidos en la investigación y proponiendo futuras líneas de investigación.

Para la recogida de datos se utilizaron diferentes técnicas cuantitativas y cualitativas, con el objetivo de cubrir tanto los aspectos empíricos y cuantificables, como los no numéricos (opiniones, comportamientos, etc.) (Cohen y Manion, 2004; Nunan, 1992). Algunos ejemplos de las primeras son un test de inteligencia verbal, un pre-test de conocimiento de la lengua inglesa, pruebas objetivas y un post-test. En cuanto a las técnicas cualitativas, se completaron diversos cuestionarios, encuestas, entrevistas, diarios de aprendizaje, etc. En la tabla 1 se ofrece un resumen completo de los instrumentos de medida utilizados.

Tabla 1. Técnicas cuantitativas y cualitativas utilizadas.

\begin{tabular}{|l|l|}
\hline \multicolumn{1}{|c|}{ Técnicas cuantitativas } & \multicolumn{1}{c|}{ Técnicas cualitativas } \\
\hline Test inteligencia verbal & Cuestionario nivel socio-económico \\
\hline Pre-test nivel conocimiento lengua inglesa & Cuestionario uso de las TIC \\
\hline $\begin{array}{l}\text { Pruebas objetivas (3 evaluaciones) } \\
-2 \text { controles por evaluación } \\
-1 \text { examen global cada evaluación }\end{array}$ & Portfolio europeo lenguas \\
\hline Examen final & $\begin{array}{l}\text { Diario de investigación } \\
\text { (investigador) }\end{array}$ \\
\cline { 2 - 3 } & $\begin{array}{l}\text { Diario de aprendizaje (sujetos } \\
\text { experimento) }\end{array}$ \\
\cline { 2 - 3 } & Observación sistemática \\
\cline { 2 - 3 } & Foro de discusión \\
\cline { 2 - 2 } & $\begin{array}{l}\text { Entrevistas post-test (grupo } \\
\text { experimental) }\end{array}$ \\
\cline { 2 - 2 } & $\begin{array}{l}\text { Cuestionario post-test (grupo } \\
\text { experimental) }\end{array}$ \\
\hline
\end{tabular}

\subsection{Contexto}

El experimento se desarrolló en el Colegio Los Tilos, un centro privado concertado en el distrito de Puente de Vallecas, en el sureste de Madrid. Se trata de un colegio de educación diferenciada, con alumnado exclusivamente de sexo femenino, cuyo perfil sociológico es en su mayoría de clase media-baja y de nacionalidad española; el porcentaje de alumnado inmigrante es muy bajo, en su mayoría procedente de países hispanohablantes. Comprende todos los niveles educativos desde Educación Infantil hasta Bachillerato y en 
$2^{\circ}$ Bachillerato, el nivel elegido para el experimento, había 16 alumnos, ocho por clase, durante ese curso escolar.

\subsection{Variables e instrumentos de medida aplicados al experimento}

Bien es sabido que en cualquier estudio estadístico son consideradas variables las características de cada sujeto en una base de datos, denominadas así porque varían de sujeto a sujeto (Nunan, 1992). En la presente investigación se han analizado tres tipos de variables (Tejedor, 1994): variable independiente, variable dependiente y variables extrañas, para las que se han combinado instrumentos de medida cualitativos y cuantitativos, tal y como se ha señalado con anterioridad:

1) Variable independiente, o variable manipulada para estudiar sus efectos sobre la variable dependiente. En este caso se trata del uso del aula virtual de inglés con el grupo experimental.

2) Variables dependientes son los elementos sobre los que influye la aplicación del método experimental. Dentro de las variables dependientes ha de distinguirse la variable principal de las secundarias. En este caso la variable principal es la posible mejora del nivel de lengua inglesa -especialmente la comprensión y expresión escrita- y las variables secundarias el aumento del tiempo dedicado a la asignatura y el aumento de la motivación a la hora de estudiar esta lengua extranjera (véase tabla 2 a continuación):

Tabla 2. Variables dependientes e instrumentos de medida.

\begin{tabular}{|l|l|}
\hline \multicolumn{1}{|c|}{ Variables dependientes } & \multicolumn{1}{c|}{ Instrumentos de medida } \\
\hline - Mejora del nivel de lengua inglesa (especialmente & - Test de nivel PET \\
comprensión y expresión escrita) & - Calificaciones en las evaluaciones \\
- Aumento del tiempo dedicado a la asignatura & $(3)$ \\
- Aumento de la motivación & - Calificación final del curso \\
& - Estadísticas del aula virtual \\
& - Cuestionario \\
\hline
\end{tabular}

La variable principal, al ser cuantificable, ha sido la de mayor importancia en el análisis de los datos obtenidos. Las otras dos variables (aumento de tiempo dedicado a la asignatura y aumento de la motivación) han aportado información cualitativa al análisis, pero no posibilitan conclusiones definitivas, por lo que se han tenido en cuenta en menor medida para evitar debilitar la validez interna de la investigación (Cohen y Manion, 2004; Nunan, 1992).

3) Variables extrañas son aquellos elementos que pueden producir un efecto sobre la variable dependiente y afectar la validez interna de la investigación, pero no son de interés para el experimento en sí: sexo, edad, y otros datos que ayudan a configurar el perfil de los sujetos participantes en el experimento, tal y como se observa en la tabla 3: 
Tabla 3. Variables extrañas e instrumentos de medida.

\begin{tabular}{|l|l|}
\hline \multicolumn{2}{|c|}{ Variables extrañas } \\
\hline Sexo & Ficha personal \\
\hline Edad & Ficha personal \\
\hline Nivel cultural de los padres & Cuestionario socio-económico \\
\hline Nivel socio-económico & Cuestionario socio-económico \\
\hline $\begin{array}{l}\text { Nivel de uso y conocimiento de las nuevas } \\
\text { tecnologías }\end{array}$ & Cuestionario uso de las TIC \\
\hline Inteligencia verbal & Test de aptitudes escolares \\
\hline Condición de repetidor & Ficha personal \\
\hline Calificación del curso anterior & Ficha personal \\
\hline
\end{tabular}

\subsection{Análisis e interpretación de los datos obtenidos}

El análisis exploratorio de datos recogidos ha sido realizado en cinco etapas, como corresponde a este tipo de investigación (Figueras y Gargallo, 2003):

1. Preparación de los datos para hacerlos accesibles a cualquier técnica estadística.

2. Examen gráfico de la naturaleza de las variables individuales a analizar y análisis descriptivo numérico que permita cuantificar algunos aspectos gráficos de los datos.

3. Examen gráfico de las relaciones entre las variables analizadas y análisis descriptivo numérico que cuantifique el grado de interrelación entre ellas.

4. Identificación de casos atípicos (outliers) y evaluación del impacto potencial que puedan tener.

5. Identificación de datos ausentes (missing) y evaluación del impacto potencial que puedan tener.

De este modo se ha podido realizar un análisis descriptivo exhaustivo de las variables dependientes y extrañas, previa obtención de tablas de frecuencias que nos ofrecieran los datos de forma global y visual. Las variables extrañas nos ayudan a trazar un perfil del grupo a estudiar. Se trata de un grupo de jóvenes entre 16 y 18 años, de sexo femenino, que pertenecen a la clase media-baja (casi un tercio, 68,7\%, perciben ayudas para el estudio). Prácticamente la totalidad de sus padres trabajan (93,9\%), mientras que solamente la mitad de las madres $(56,3 \%)$ se ha incorporado al mundo laboral (el porcentaje es algo superior en las madres del grupo de control que en las del grupo experimental). El nivel cultural de los progenitores es medio-alto, ya que un $62,5 \%$ poseen estudios superiores y sus hábitos de lectura son bastante positivos, pues incluyen la lectura habitual de prensa $(100 \%$ de los encuestados) y el préstamo de libros en bibliotecas públicas (56,2\%). Es un grupo totalmente familiarizado con las nuevas tecnologías y dispositivos TIC, que dispone de todo tipo de avances tecnológicos en sus hogares (televisión con pantalla plana, vídeo, DVD, MP3, teléfono móvil, PC y / o portátil). Todos los sujetos cuentan con conexión a Internet tipo ADSL y suelen utilizar el ordenador e Internet a diario. Son en su mayoría autodidactas, forman parte de la generación de nativos digitales (Prensky, 2001) y no tienen problemas 
para realizar las tareas informáticas comunes relacionadas con la ofimática y las actividades básicas a nivel usuario (instalación de dispositivos periféricos, copia y traslado de ficheros, utilización del procesador de textos). Consideran tanto el ordenador como Internet una de sus formas de ocio, más que una herramienta para el trabajo escolar, y sus actividades favoritas son escuchar música, ver vídeos o jugar a juegos de ordenador.

En cuanto al rendimiento académico, se muestran en la franja de coeficiente intelectual normal, siendo el del grupo experimental $(103,75)$ ligeramente superior al del grupo de control $(99,25)$. Hay dos sujetos repetidores, ambos en el grupo de control (aparte de otros dos que han repetido cursos anteriores), y las calificaciones obtenidas en la asignatura de lengua inglesa en el curso anterior muestran que el grupo experimental es mejor en esta disciplina que el grupo de control: el primero alcanzó una media de 7,50 en $1^{\circ}$ Bachillerato, mientras que la media del segundo está casi un punto por debajo $(6,63)$.

Todos estos elementos hacen ver que los grupos no son exactamente iguales, por lo que además del estudio inter-grupos se hace necesario un estudio intra-grupo, comparando a cada grupo consigo mismo. Es conveniente recordar en este punto que el tipo de investigación ha sido IA: por ser grupos ya establecidos, no ha sido posible reorganizarlos de manera aleatoria.

\subsubsection{Estudio inter-grupos}

En el pre-test el grupo de control obtuvo un 5,75 de media, frente al 6,25 del grupo experimental, que se sitúa medio punto por encima (véase la tabla 4). Al estudiar las medias del grupo de control y el grupo experimental en el pre-test y teniendo en cuenta los datos referidos al rendimiento académico ofrecidos por las variables extrañas se observa que no es posible hacer una comparación inter-grupos, ya que no son equiparables. Sin embargo, sí que es posible hacer una comparación intra-grupo y ver cómo se ha comportado cada grupo con respecto a sí mismo, analizando su evolución a lo largo del experimento.

Tabla 4. Medias de los grupos de control y experimental.

\begin{tabular}{|c|c|c|c|c|c|}
\hline Grupos & $\begin{array}{c}\text { Pre-test } \\
\text { Sept. } 08\end{array}$ & $\begin{array}{c}1^{\text {a }} \text { Eval } \\
\text { Nov. } 08\end{array}$ & $\begin{array}{c}2^{\text {a }} \text { Eval } \\
\text { Feb. 09 }\end{array}$ & $\begin{array}{c}3^{\text {a }} \text { Eval } \\
\text { Abr. 09 }\end{array}$ & $\begin{array}{c}\text { Post-test } \\
\text { May. 09 }\end{array}$ \\
\hline Control & 5,75 & 5,38 & 5,13 & 6 & 6,13 \\
\hline Experimental & 6,25 & 6,63 & 6,75 & 6,63 & 7,63 \\
\hline Ambos grupos & 6 & 6,005 & 5,94 & 6,315 & 6,88 \\
\hline
\end{tabular}

\subsubsection{Estudio intra-grupo}

El grupo de control bajó su rendimiento desde el pre-test hasta la segunda evaluación, y después mejoró sus resultados, consiguiendo superar los valores iniciales en la tercera evaluación y en el post-test. Sin embargo, las ganancias cuantitativas del grupo de control pueden ser calificadas como discretas, pues mejora menos de medio punto entre el comienzo y el final del experimento $(+0,38)$, o lo que es lo mismo, incrementa su media poco más de un $6 \%(6,61 \%$ exactamente), puesto que partía de un 5,75 y la nota final en el post-test 
es 6,13 . Por tanto, la conclusión alcanzada tras el estudio intra-grupo del grupo de control es la siguiente:

El grupo de control, que ha continuado con la metodología tradicional, apenas ha mejorado a lo largo de $2^{\circ}$ Bachillerato (solamente un 6,61\%).

Sin embargo, el grupo experimental muestra desde el comienzo un aumento en los resultados con respecto al pre-test. Ya en la primera evaluación es evidente su progreso, con un aumento de $+0,38$ puntos con respecto al pre-test, exactamente los mismos puntos que subirá el grupo de control al final del experimento. Se puede afirmar que el grupo experimental, con la metodología innovadora, ha mejorado en un trimestre lo que el grupo de control tardará en lograr todo un curso escolar. Se ha producido, por tanto, una aceleración en el progreso y aprendizaje en el grupo experimental gracias al uso del aula virtual. Esta tendencia alcista continúa en el grupo experimental durante todo el curso, el periodo coincidente con la duración del experimento, y en el post-test obtiene una nota media muy por encima de los resultados del pre-test $(+1,38)$, ya que partía de un 6,25 y obtiene un 7,63 en el post-test (en la tabla 5 se desglosan con detalle todos estos resultados y en la figura 3 se puede apreciar la evolución de forma gráfica). Así pues, la conclusión a la que se llega después de estudiar la evolución del grupo experimental es la siguiente:

El grupo que disfruta del método innovador tiene unas ganancias cuantitativas de más del $20 \%(22,08 \%)$, un porcentaje muy significativo que corrobora la eficacia del uso de EVA en la enseñanza del inglés.

Tabla 5. Comparativa del pre-test, evaluaciones y post-test.

\begin{tabular}{|l|r|r|r|r|}
\hline \multicolumn{1}{|c|}{ Grupos } & $\begin{array}{c}\mathbf{1}^{\text {a }} \begin{array}{c}\text { Eval. vs Pre-test } \\
\text { (puntos) }\end{array} \\
\text { Control }\end{array} \quad-0,37$ & $\begin{array}{c}\mathbf{2}^{\mathrm{a}} \text { Eval. vs Pre-test } \\
\text { (puntos) }\end{array}$ & $\begin{array}{c}3^{\mathrm{a}} \text { Eval. vs Pre-test } \\
\text { (puntos) }\end{array}$ & $\begin{array}{c}\text { Post-test vs Pre-test } \\
\text { (puntos) }\end{array}$ \\
\hline Experimental & $+0,38$ & $-0,62$ & $+0,25$ & $+0,38$ \\
\hline
\end{tabular}

\begin{tabular}{|l|r|r|r|r|}
\hline \multicolumn{1}{|c|}{ Grupos } & $\begin{array}{c}1^{\mathrm{a}} \text { Eval. vs Pre-test } \\
(\%)\end{array}$ & $\begin{array}{c}2^{\mathrm{a}} \text { Eval. vs Pre-test } \\
(\%)\end{array}$ & $\begin{array}{c}3^{\mathrm{a}} \text { Eval. vs Pre- } \\
\text { test }(\%)\end{array}$ & $\begin{array}{c}\text { Post-test vs Pre- } \\
\text { test }(\%)\end{array}$ \\
\hline Control & $-6,43 \%$ & $-10,78 \%$ & $+4,35 \%$ & $+6,61 \%$ \\
\hline Experimental & $+6,08 \%$ & $+8,00 \%$ & $+6,08 \%$ & $+22,08 \%$ \\
\hline
\end{tabular}

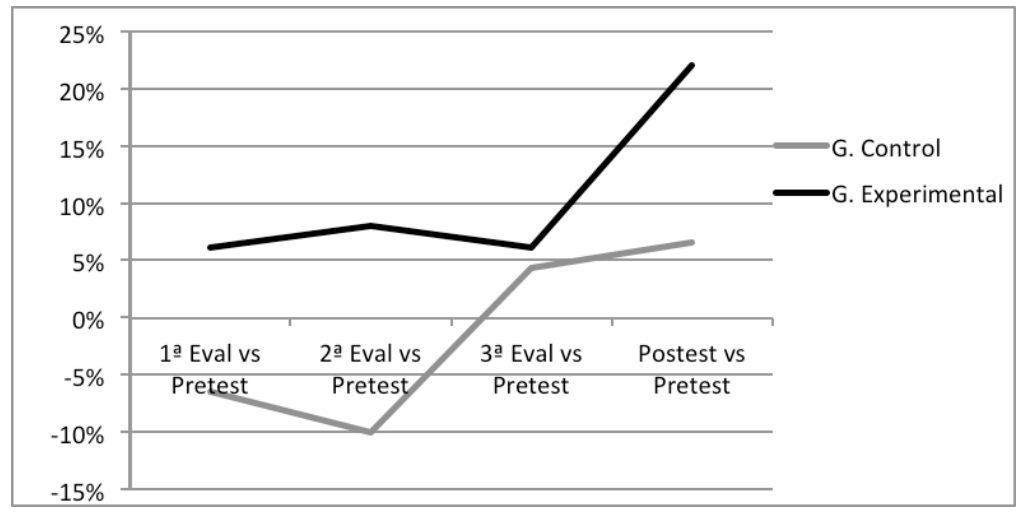

Figura 3.

Evolución de los resultados comparados con el pre-test. 


\section{Resultados y CONCLUSiones}

En vista de los resultados analizados e interpretados, se comprueba que la hipótesis de trabajo, la mejora en los resultados por parte del grupo experimental que ha utilizado la metodología innovadora, se confirma: hay una evidente mejora en los resultados académicos de este grupo, la cual demuestra que el uso del aula virtual como plataforma de apoyo en la preparación de la PAU de lengua inglesa es efectivo para mejorar la comprensión y la expresión escrita, únicos componentes que son evaluados en la PAU vigente en el momento del experimento. Por lo tanto, se puede afirmar que, según los resultados cuantitativos y cualitativos ofrecidos por el experimento:

El uso de los EVA es eficaz para el progreso de la comprensión y la expresión escrita en la preparación de la PAU en lengua inglesa y permite la mejora de los resultados académicos por parte del alumnado.

Así pues, tras finalizar la investigación se puede concluir que:

La preparación de la PAU de inglés como lengua extranjera con el apoyo del aula virtual de Moodle es beneficiosa para el alumnado, pues aumenta el tiempo de dedicación a la asignatura, su motivación a la hora de estudiar inglés y, sobre todo, repercute de manera favorable en sus resultados académicos.

Además, una vez terminada la investigación, el grupo experimental siguió utilizando el aula virtual para la preparación de la PAU, que tuvo lugar un mes después de la finalización del experimento, y aunque este examen se halla fuera del contexto de la investigación en sí, la autora quiere dejar aquí constancia de los resultados obtenidos por los sujetos del grupo de control y experimental. El grupo de control obtuvo una media de 4,41, que sería un suspenso (en el post-test llegó a 6,13) mientras que el grupo experimental alcanzó una media de 7,20, nota muy similar a la obtenida en el post-test $(7,63)$. Resulta aquí evidente el papel que juega el aula virtual como herramienta de apoyo en la PAU: los alumnos del grupo experimental, motivados por la utilización de esta metodología innovadora, siguieron practicando las actividades en línea diseñadas para la preparación de la PAU, mientras que el grupo de control, que disponía de los materiales impresos característicos de la metodología tradicional, probablemente se vio menos motivado para volver sobre ellos y repasar; esto explicaría el descenso en su rendimiento en la PAU de inglés.

En el siguiente curso escolar, 2009-2010, esta metodología innovadora se hizo extensiva a todos los grupos de Bachillerato y la media del centro en la prueba de lengua extranjera mejoró notablemente, hasta un 8,5 , una calificación altamente satisfactoria que corrobora la eficacia del Aula Virtual de Inglés para la preparación de la PAU.

El uso de EVA en la enseñanza de idiomas está ya muy extendido entre la comunidad educativa; sin embargo, aunque ha habido algunos intentos (Brandl, 2005; Ezeiza, Fernández Marzo y Pérez Manso, 2010), la investigación sobre Moodle como plataforma para la enseñanza de idiomas ha sido escasa y principalmente se ha llevado a cabo en el contexto de la educación universitaria, no en las enseñanzas medias. Por esta razón, la autora considera que el presente estudio supone una contribución de interés en el campo de la ELAO y los EVA, más concretamente en Moodle, ya que se ha diseñado un modelo para el uso de esta plataforma con la metodología de blended learning, de manera que contribuya a la mejor preparación del alumnado para un examen tan importante como la PAU. No es solamente interesante el haber confirmado los beneficios del uso del aula virtual tanto en la mejora de 
resultados como en el aumento de la motivación y el tiempo dedicado a la asignatura, sino que además se ha demostrado la versatilidad de Moodle y se han puesto en evidencia las múltiples posibilidades didácticas de esta herramienta.

La autora ha continuado profundizando en este campo en los meses posteriores al experimento, centrándose en las profundas transformaciones que han de producirse en la PAU en lengua extranjera tras la implantación de la Ley Orgánica de Enseñanza, que espera incorporar una prueba oral. El software de Moodle puede facilitar la implementación de dicha prueba y la línea de investigación adoptada apunta en esa dirección: la utilización de estos EVA con la finalidad de diseñar una prueba informatizada para la PAUen lengua extranjera a la altura de pruebas similares que se realizan en otros países, aprovechando las ventajas que ofrecen las plataformas para la ELAO, siempre con el transfondo metodológico del MCER de manera que se cubran todas las competencias en él descritas, incluyendo la parte oral, y se mantenga un enfoque orientado a la acción.

\section{REFERENCIAS BIBLIOGRÁFICAS}

Altrichter, H., Posch, P. y Somekh, B. (1993). Teachers investigate their work. Londres: Routledge.

Barrett, B. \& Sharma, P. (2007). Blended Learning. Oxford: Macmillan.

Blake, N. y Standish, P. (2000). Enquiries at the interface: Philosophical problems of online education. Oxford: Blackwell.

Brandl, K. (2005). "Are you ready to "Moodle"?", in Language Learning \& Technology, 9, 2, 16-23. Disponible en http://1lt.msu.edu/vol9num2/pdf/review1.pdf. Acceso 21 de noviembre de 2011.

Buendía, L. et al. (ed.) (1999). Modelos de análisis de la investigación educativa. Sevilla: Ediciones Alfar.

Cabero, J. (2006). "Comunidades virtuales para el aprendizaje. Su utilización en la enseñanza". EDUTEC, Revista Electrónica de Tecnología Educativa, 20. Disponible en http://edutec. rediris.es/Revelec2/ revelec20/ cabero20.htm. Acceso 18 de noviembre de 2011.

Carretero, M. (1993). Constructivismo y educación. Madrid: Edelvives.

Chapelle, C. (2007). Towards Adaptive CALL. Disponible en http://www.public.iastate.edu/ apling/ TSLL/5th_2007/ proceedings2007/contents.html. Acceso 21 de noviembre de 2011.

Claypole, M. (2010). Controversies in ELT. Norderstedt: LinguaBooks.

Cohen, L. y Manion, L. (2004). Research methods in education. Londres y Nueva York: Routledge.

Consejo de Europa. (2002). Marco común europeo de referencia para las lenguas: Aprendizaje, enseñanza, evaluación. Madrid: MECD-Anaya.

Dougiamas, M. (1998). A journey into Constructivism. Disponible en http://dougiamas.com/writing/ constructivism.html. Acceso 19 de noviembre de 2011.

Ducajú, M. (2009, 12 de mayo). Los estudiantes llegarán a la prueba de selectivo de inglés sin dominar el idioma. Levante - El Mercantil Valenciano. Disponible en http://www.levanteemv.com/secciones/noticia.jsp?pRef $=$ 2009051200_19_588493_Comunitat-Valencianaestudiantes-llegaran-prueba-selectivo-ingles-dominar-idioma. Acceso 10 de noviembre de 2011.

Elliot, J. (1997). La investigación-acción en educación. Madrid: Morata. 
Estadísticas de Selectividad Junio 2006. (2008). Disponible en http://www.emes.es/AccesoUniversi$\mathrm{dad} /$ Selectividad/Estadisticas/tabid/427/Default.aspx. Acceso 11 de noviembre de 2011.

Ezeiza, A., Fernández Marzo, F. y Pérez Manso, A. (2010). Moodle en la enseñanza de lenguas: el proyecto COVCELL. Disponible en www.ainhoaezeiza.net/file.php/1/argitalpenak/ Desarrollo_de_moodle_ensen_lenguas_proyecto_covcell.pdf. Acceso 10 de febrero de 2011.

Fernández López, S. (2003). Propuesta curricular y Marco común europeo de referencia: desarrollo por tareas. Madrid: Edinumen.

Figueras, M.S. y Gargallo, P. (2003). Análisis exploratorio de datos. Disponible en http://ciberconta.unizar.es/Leccion/ aed/inicio.html. Acceso 21 de noviembre de 2011.

Hanson-Smith, E. (2003). "A Brief history of CALL theory", in CATESOL Journal, 15 (1): 2130.

Larsen-Freeman, D. y Long, M. (1991). An introduction to second language acquisition research. Londres: Longman.

Latorre, A. (2003). La investigación-acción. Conocer y cambiar la práctica educativa. Barcelona: Graó.

Latorre, A., Rincón, D. y Arnal, J. (1996). Bases metodológicas de la investigación educativa. Barcelona: Graó.

Levy, M. y Stockwell, G. (2006). CALL dimensions: Options and issues in computer-assisted language learning. Mahwah, NJ: Lawrence Erlbaum.

Ley Orgánica 1/1990, de 3 de octubre, de Ordenación General del Sistema Educativo (LOGSE). (BOE núm. 238, de 4 de octubre de 1990).

Ley Orgánica 2/2006, de 3 de mayo, de Educación (LOE). (BOE núm. 106, de 4 de mayo de 2006).

Masters, J. (1995). "The History of Action Research". En Hughes, I. (ed.), Action research electronic reader. Disponible en http://www.scu.edu.au/schools/gcm/ar/arr/arow/rmasters.html. Acceso 17 de noviembre de 2011.

Muñoz, M. (2009, 6 de mayo). "El inglés estropea los buenos resultados de los alumnos riojanos en Selectividad", en El Correo Digital, Disponible en http://www.elcorreodigital.com/ vizcaya/20090506/rioja/ingles-estropea-buenos-resultados-20090506.html. Acceso $11 \mathrm{de}$ noviembre de 2011.

Neumeier, P. (2005). "A closer look at blended learning - parameters for designing a blended learning environment for language teaching and learning", in ReCALL: the Journal of EUROCALL, 17(2): 163-178.

Nunan, D. (1992). Research methods in language learning. Cambridge: Cambridge University Press.

Nunan, D. (2000). Language teaching methodology. Harlow: Longman.

Prensky, M. (2001). "Digital Natives, Digital Immigrants", in On the Horizon, Vol. 9 (5). Disponible en http://www.marcprensky.com/writing/Prensky\%20-\%20Digital\%20Natives, $\% 20$ Digital\%20Immigrants\%20-\%20Part1.pdf. Acceso 21 de noviembre de 2011.

Palacios, I. (Dir.) (2007). Diccionario de enseñanza y aprendizaje de lenguas. Madrid: EnclaveELE / CLE International.

Salinas, J. (2008). Comunidades virtuales y aprendizaje digital. Disponible en http://www.colombiaaprende.edu.co/ html/mediateca/1607/article-173269.html. Acceso 24 de noviembre de 2011.

Sánchez Pérez, A. (2000). Los métodos en la enseñanza de idiomas. Evolución histórica y análisis didáctico. Madrid: SGEL. 
Sharma, P. (2010). "Blended learning", in ELT Journal, 64 (4): 456-458.

Suárez, M. (2002). "Algunas reflexiones sobre la investigación-acción colaboradora en educación”, en Revista Electrónica de Enseñanza de las Ciencias, 1, 1. Disponible en http://reec.uvigo. es/volumenes/volumen1/Numero1/Art3.pdf. Acceso 17 de noviembre de 2011.

Richards, J.C. y Rodgers, T.S. (2001). Approaches and methods in language teaching. Nueva York: Cambridge University Press.

Tejedor, F. (1994). "La experimentación como método de investigación educativa", en V. García Hoz (dir.), Problemas y métodos de investigación en educación personalizada. Madrid: Rialp, 256-285.

Varela, R. (Coord.). (2003). All about teaching English. Madrid: CERASA. 